\title{
Nighttime low illumination image enhancement with single image using bright/dark channel prior
}

\author{
Zhenghao Shi $^{1 *}$, Mei mei Zhu ${ }^{1}$, Bin Guo ${ }^{2}$, Minghua Zhao ${ }^{1}$ and Changqing Zhang ${ }^{3}$
}

\begin{abstract}
Nighttime low illumination image enhancement is highly desired for outdoor computer vision applications. However, few works have been studied towards this goal. In addition, the low illumination enhancement problem becomes very challenging when the depth information of a low illumination image is unknown. To address this problem, in this paper, we propose a dual channel prior-based method for nighttime low illumination image enhancement with a single image, which builds upon two existing image priors: dark channel prior and bright channel prior. We utilize the bright channel prior to get an initial transmission estimate and then use the dark channel as a complementary channel to correct potentially erroneous transmission estimates attained from the bright channel prior. Experimental results show significant credibility of the approach both visually and by quantitative comparison with existing methods.
\end{abstract}

Keywords: Nighttime illumination, Image enhancement, Dark channel prior, Bright channel prior

\section{Introduction}

Images captured in nighttime low illumination usually suffer from a poor visibility (as shown in Fig. 1), and has negatively impacts on subsequent processing for outdoor computer vision applications [1]. Thereby, to ensure the reliable of outdoor vision systems under nighttime, it is highly desirable to enhance the visibility of low illumination images.

However, due to the unknown depth information of a low illumination image, nighttime low illumination enhancement is a challenging problem. Therefore, numerous efforts have been made to address this problem in past years. One of the most common used methods is using multiple images or additional information for image enhancement. For example, images taken at different times are combined to enhance nighttime images in $[2,3]$; infrared images are fused with visible images for the enhancement of nighttime images in $[4,5]$. The basic idea of such methods is to exploit the advantages of multiple images by using image fusion techniques. Though this

*Correspondence: zhenghaoshimtap@163.com

'School of Computer Science and Engineering, Xi'an University of Technology, No.5 Jinhua South Road, 710048 Xi'an, China

Full list of author information is available at the end of the article kind of method can enhance low illumination night-time image effectively, it requires exact point-to-point fusion between different images, which is not always available.

Compared to using multiple images for image enhancement, image enhancement with a single image does not need additional assistant images and has received increasing interests recently. For example, aiming at contrast enhancement, many conventional image processing techniques or their modifications, such as the well-known histogram equalization-based methods [6-9] and wavelet transform-based method [10], are widely employed to enhance the darks at the cost of saturating bright. In order to avoid structure information loss, retinex-based methods, including single-scale retinex (SSR), multiple scale-retinex (MSR)-based methods [11, 12], and the MSR with color restoration (MSRCR) [13], were employed to suppress noise and preserve highlighted regions of the original image. To obtain better color restoration, sparse representations of low illumination image patches in an appropriate dictionary was used to approximate the corresponding daytime images in [14] and [15], respectively. However, though these methods could enhance low illumination images at a certain extent, they might also lead to contrast over-enhancement or noise amplification (as shown in Fig. 2) due to constrains of stretching range in 

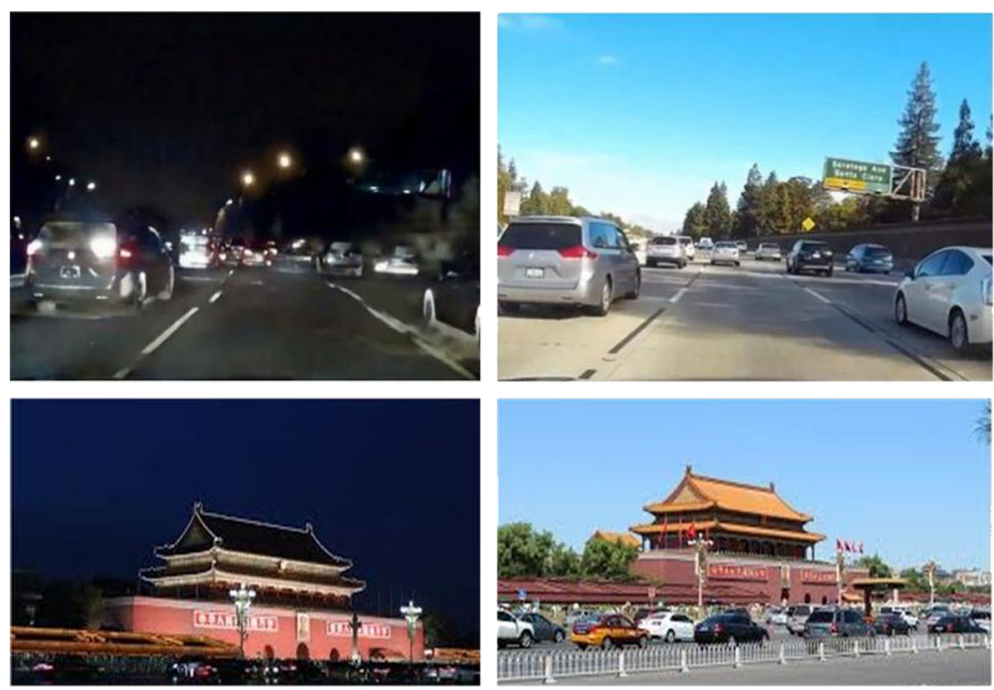

a

b

Fig. 1 Images captured in nighttime low illumination and daytime. a Images captured in nighttime. b Images captured in daytime

the solving model, or difficulty of designing of parameters, and well solutions for the problems are still opened.

Based on two existing image priors-dark channel prior and bright channel prior-this paper proposes a dual channel prior-based method for low illumination image enhancement with a single image. The contributions of the proposed method are mainly in two aspects:

1. We propose an accuracy transmission estimation method based on bright channel prior and dark channel prior. We firstly utilize the bright channel prior to get an initial transmission estimation, and then use the dark channel as a complementary channel to correct potentially erroneous transmission estimates attained from the bright channel prior.

2. We develop an effective method for low illumination image enhancement with a single image based on bright channel prior and dark channel prior.

Experimental results demonstrate that the proposed method is very effective in enhancing low-light images and outperforms state-of-the-art ones in contrast enhancement.
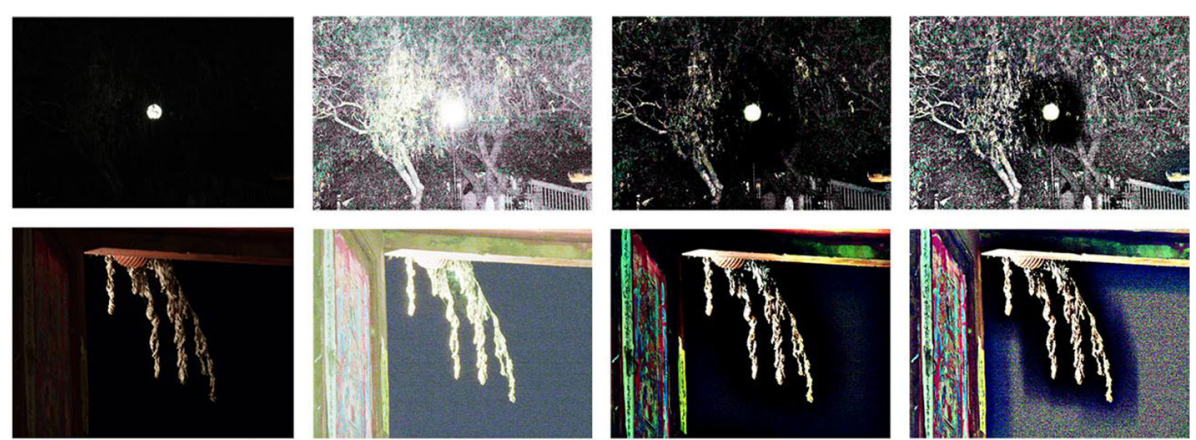

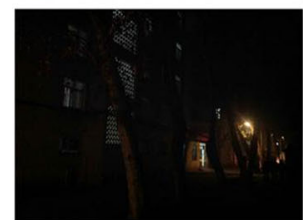

a

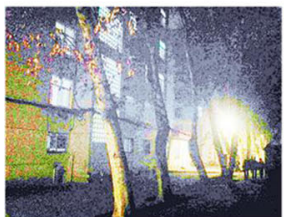

b

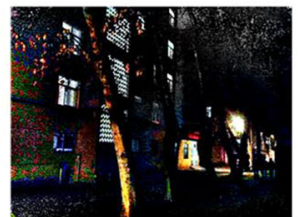

C

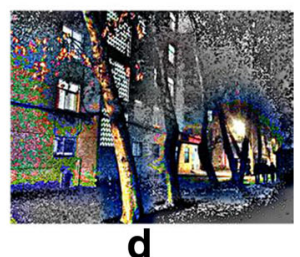

Fig. 2 Nighttime image enhancement results with different methods. a Input image. b Enhanced image with histogram equalization [8] c Enhanced image with single-scale retinex (SSR) [11]. d Enhanced image with multi-scale retinex (MSR) [12] 
The rest of this paper is organized as follows. Section 2 describes the related work in details. Section 3 presents the basic principle of dark channel prior and bright channel prior. Section 4 presents the proposed method in details. Section 5 gives the implementation of our experiments and reports the results. A further discussion is give in Section 6, and we conclude our work in Section 7.

\section{Related works}

There are many prior-based methods for single low illumination image enhancement being developed recently. One of the most famous prior is the dark channel prior [16] which have been employed for adaptively improving the contrast of images under low luminance $[17,18]$. A similar method was also developed in [19] where the luminance map is used to estimate the global atmospheric light and the transmittance according to the observed similarity between the luminance map and dark channel prior. Inspired by photographic negative imaging techniques, in our prior work [20], we also employ the dark channel prior as a photographic imaging tool for low illumination image enhancement. Although the image enhancing results with dark channel prior are visually compelling, the method may not be physically valid. A limitation of such methods is that there are boxes around where either bright spot exist or the scene depth is not continuous in resulting image.

Inspired by dark channel prior, a new image prior, bright channel prior, is proposed to estimate local exposure for under exposed images in [21] and [22], respectively. A similar method was also employed in [23], where they estimate the illumination of an input image using a two-stage map method; the illumination of each pixel is first estimated individually by finding the maximum value in $R, G$, and $B$ channels. Further, refine the initial illumination map by imposing a structure prior on it as the final illumination map. Having the well-constructed illumination map, the enhancement can be achieved accordingly. Although this kind of methods is effective for very low illumination image, they are sensitive to bright light. If there is light object in the low illumination image or bright objects, the method might fail to obtain good enhancement.
In order to alleviate the light/bright object problem, we try to combine the bright channel prior and dark channel prior for low illumination image enhancement. We will discuss the detail in the following section.

\section{Dark/bright channel prior}

Formally, given an image $I$, the dark channel [16] can be defined as

$$
I^{\mathrm{dark}}(x)=\min _{c \in\{r, g, b\}}\left(\min _{y \in \Omega(x)}\left(I^{c}(y)\right)\right)
$$

where $I^{c}$ is a color channel of image $I$ and $\Omega(x)$ is a local patch centered at $x . y$ is a pixel in the local path $\Omega(x)$. According to [16], we know that for a haze-free image, $I^{\text {dark }}(x) \rightarrow 0$. For a hazed image, $I^{\text {dark }}(x)$ is no longer black. In hazed images, the intensity of the dark pixels in that channel is mainly contributed by the airlight. Therefore, these dark pixels can directly provide accurate estimation of the haze's transmission.

Inspired by He's dark channel prior [16], a similar image prior was also proposed in [21], where they found that in most of good illumination image patches, at least one color channel has very high intensity at some pixels. In other words, the maximum intensity in such a patch should have a very high value. Given an image $I$, formally, there is

$$
I^{\text {bright }}(x)=\max _{c \in\{r, g, b\}}\left(\max _{y \in \Omega(x)}\left(I^{c}(y)\right)\right)
$$

where $I^{c}$ is a color channel of $I$ and $\Omega(x)$ is a local patch centered at $x . y$ is a pixel in the local path $\Omega(x)$. Further observations show that if $I$ is a good illumination image, the intensity of $I^{\text {bright }}(x)$ is a very limited high intensity value and mostly tends to be 255 . Similar to He's dark channel prior, they termed $I^{\text {bright }}(x)$ as the bright channel of $I$ and called the above knowledge as the bright channel prior.

Figure 3 shows an example of the dark channel and bright channel for a same scene; the local patch size for both operation was set as $15 \times 15$. From Fig. 3, it can be seen that image structure in the bright channel is more complete than that in the dark channel, while some missed

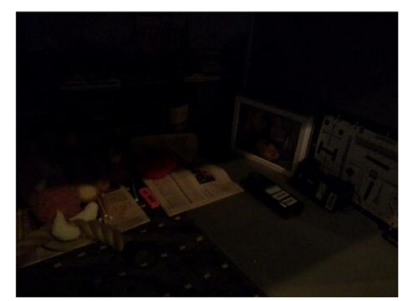

a

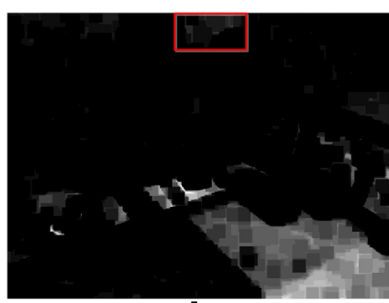

b

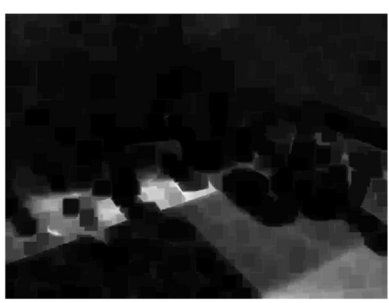

C

Fig. 3 An example of the dark channel and bright channel. a Input image. b Dark channel. c Bright channel 
structures in the bright channel appear in the dark channel, as indexed by the red rectangle in Fig. 3b. Therefore, we can use the dark channel as a complementary to correct potentially erroneous transmission estimates attained from the bright channel prior.

\section{Proposed method}

4.1 Overview of the proposed method

Given an input nighttime low illumination image I, its image exposure model can be represented as

$$
I(x)=J(x) t(x)+A(1-t(x))
$$

where $J$ is the scene radiance (viz., the well exposed image), $A$ is the global atmospheric light, and $t$ is the medium transmission describing the portion of the light that is not scattered and reaches the camera.

Our aim is to correct the low illumination image $I$ and get the well exposed image $J$. From Eq. (3), we can get

$$
J(x)=\frac{I(x)-A}{t(x)}+A
$$

From Eq. (4), it is obviously that if $t(x)$ and $A$ are known, then we can easily obtain the well exposed image $J$.

Figure 4 shows the framework of the proposed method, it begins from the computation of the dark channel and the bright channel of the input image. The basic operation steps are the following: (1) Firstly, we use a max filter and a min filter to obtain the bright channel and the dark channel of input image, respectively. (2) Then, we compute the global atmosphere light via a min filtering operation on the bright channel. (3) Next, we utilize the bright channel prior to get an initial transmission estimation. (4) Then, we use the dark channel as a complementary information to correct potentially erroneous transmission estimations attained from the bright channel prior. (5) The corrected transmission map is refined via the guided filter to get more smooth structure. (6) Finally, with the global atmosphere light and transmission, we can achieve a dark-free image. From afore-mentioned, details of each key operation are described in the following sections.

\subsection{Atmospheric light estimation}

For atmospheric light estimation, a similar method to that used in [16] was used in this paper.

Firstly, we pick the top $10 \%$ brightest pixels in the bright channel. As shown in Fig. 3c, the bright channel approximates the dark denseness of the atmosphere of the input image very well, and therefore, we can use the bright channel to improve the atmospheric light estimation.

And then the pixels with the mean intensity of these pixels in the input image $I$ is selected as the atmospheric light in an effort to avoid overestimation of the atmospheric

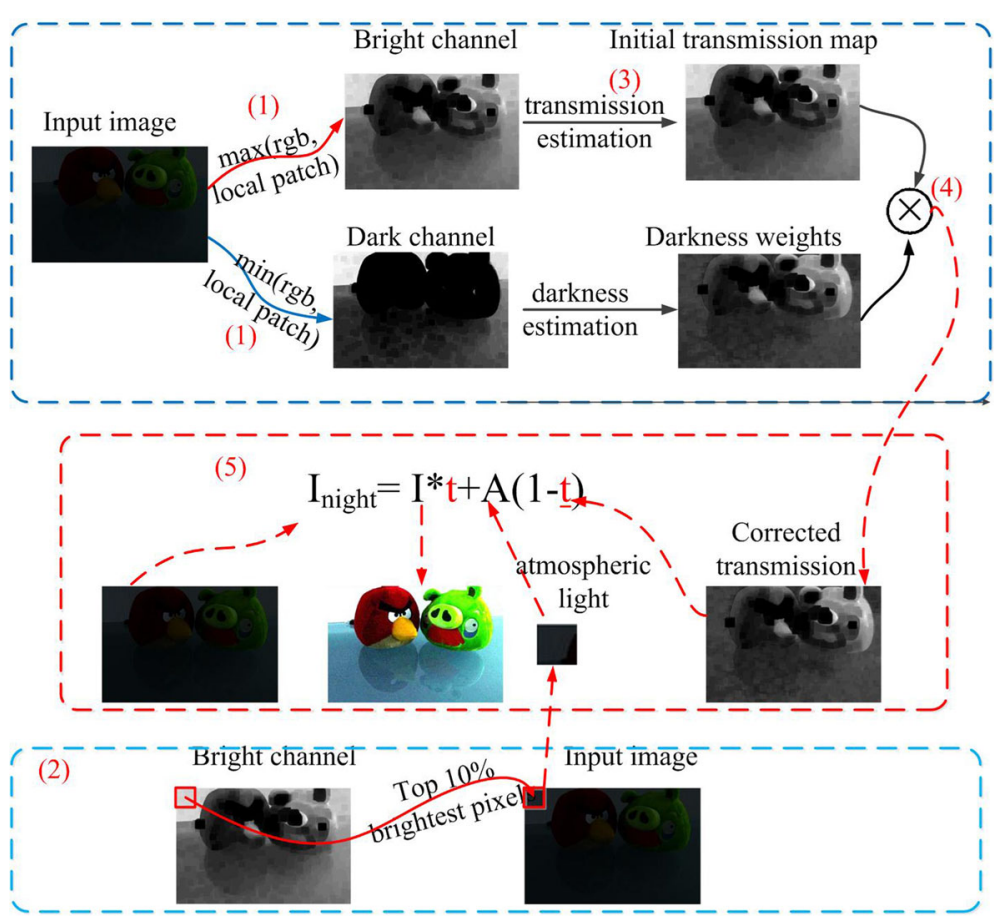

Fig. 4 Framework of the proposed method. The key of the method is to use dual channel prior for transmission estimation. We utilize the bright channel prior to get an initial transmission first, and then use the dark channel as a complementary information to correct potentially erroneous transmission estimations attained from the bright channel prior 


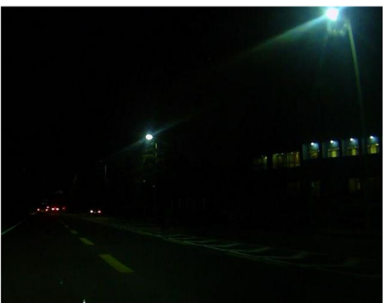

a

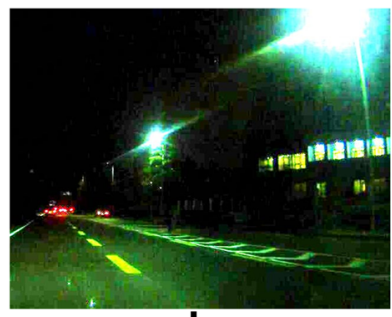

b

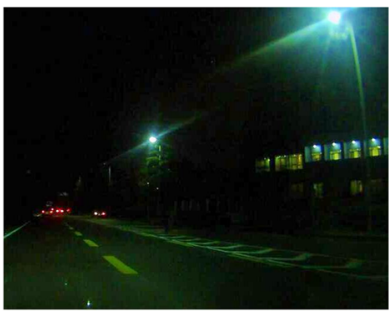

C

Fig. 5 Illustration of result selecting the "brightest pixel" as the atmospheric light. a Input image. b The bright channel.c The final enhancement image

light. For example, in a real low illumination image, if there exists brighter lights such as lamp light or moon light (as shown in Fig. 5a), the atmospheric light will not be the brightest one. In such case, if we select the "brightest pixel" as the atmospheric light, noise might be introduced; as shown in Fig. 5b, the lamp light in the image does not look very natural, and there is much noise around it. To avoid such issues, in this work, the mean intensity based on bright channel was selected as our estimation. Figure $5 \mathrm{c}$ shows the final enhancement image; it is obvious that the quality of the enhanced image is improved.

\subsection{Estimating the transmission using dark/bright channel prior}

Simply, we assume that the transmission in a small local patch $\Omega(x)$ is a constant. It is a reasonable assumption since a local patch usually contains same materials and has constant illumination. Denote the transmission as $\tilde{t}(x)$. Then, taking max operation in the local patch on the low illumination imaging Eq. (1), we have:

$$
\max _{y \in \Omega(x)}\left(I^{c}(y)\right)=\tilde{t}(x) \max _{y \in \Omega(x)}\left(J^{c}(y)\right)+(1-\tilde{t}(x)) A^{c}
$$

where $c \in\{r, g, b\}$ means that the max operation is performed on three color channels independently, viz,

$$
\begin{aligned}
\max _{c}\left(\max _{y \in \Omega(x)}\left(I^{c}(y)\right)\right)= & \tilde{t}(x) \max _{c}\left(\max _{y \in \Omega(x)}\left(J^{c}(y)\right)\right) \\
& +(1-\tilde{t}(x)) A^{c}
\end{aligned}
$$

According to Eq. (1), we rewrite Eq. (4) as

$$
I^{\text {bright }}(x)=\tilde{t}(x) J^{\text {bright }}(x)+(1-\tilde{t}(x)) A^{c}
$$

According to the bright channel prior, the bright channel $I^{\text {bright }}$ of a well illuminated radiance $I$ should tend to be 255:

$$
I^{\text {bright }}(x)=\max _{c}\left(\max _{y \in \Omega(x)}\left(I^{c}(y)\right)\right)=255
$$

From Eq. (7) and (8), we have:

$$
\frac{I^{\text {bright }}(x)}{A^{c}}=\tilde{t}(x) \frac{255}{A^{c}}+(1-\tilde{t}(x))
$$

From Eq. (9), we have:

$$
\tilde{t}(x)=\frac{I^{\text {bright }}(x)-A^{c}}{255-A^{c}}
$$

To not to be confused, we rewrite Eq. (10) as

$$
\tilde{t}(x)^{\text {bright }}=\frac{I^{\text {bright }}(x)-A^{c}}{255-A^{c}}
$$

From Eq. (11), we can estimate the initial transmission $\tilde{t}(x)$ with the bright channel prior simply.

As aforementioned in Section 3, the bright channel prior works by assuming that the atmospheric light is of a neutral color and tries to factor this neutral color out by measuring it from the bright channel. Because there will be at least one pixel, ensure a minimum value in at least one channel in most low illumination patches; this intuitively works. However, when there is a patch entirely containing a bright object, such as lamp light, the estimation will fail.

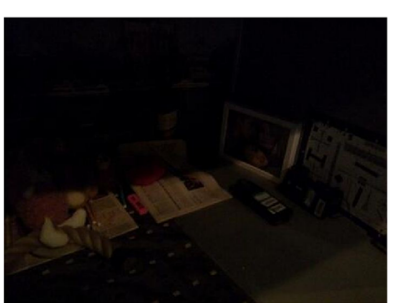

a

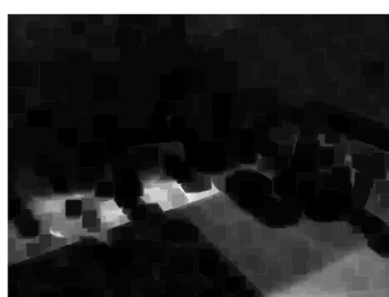

b

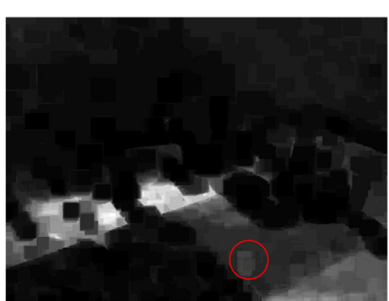

C

Fig. 6 Illustration transmission estimation result via dual channel prior. a Input image. b Transmission estimated with bright channel. c Transmission estimated with dual channel prior 


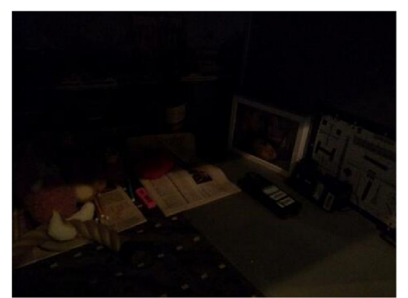

a

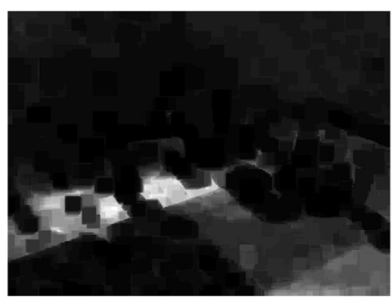

b

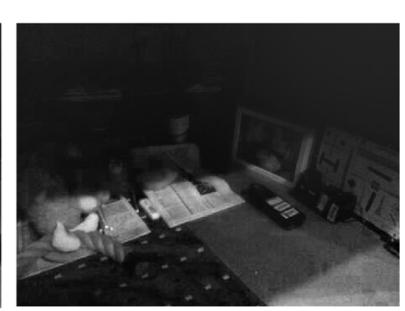

C

Fig. 7 Transmission refining result via guided filter. a Original image.b Transmission estimated with dual channel. c Transmission refined with guided filter

As shown in Fig. 5, the lamp light introduces significant bias in the final result. For regions depicting lamp light cover, the contrast between predominantly saturated dark background and high intensity lamp light introduces artificial transmission variations. Conversely, image regions containing dark objects will tend to yield artificially low values in the bright channel, causing inconsistent transmission orderings that are driven by object/region texture instead of scene nighttime properties.

To overcome the issues mentioned above, we use the dark channel as a complementary to correct potentially erroneous transmission estimates attained from the bright channel prior. Our basic idea is described as below:

Firstly, we compute the difference between the bright channel and the dark channel:

$$
I_{\text {channel }}^{\text {difference }}(x)=I^{\text {bright }}(x)-I^{\text {dark }}(x)
$$

Then, we determine whether the value of $I_{\text {channel }}^{\text {difference }}(x)$ is smaller than a predefined threshold $\alpha$ or not. The threshold $\alpha$ is determined by empirical experiment. In this work, $\alpha=0.4$. If the value of $I_{\text {channel }}^{\text {difference }}(x)$ is smaller than the predefined threshold $\alpha$, the pixel $x$ should be in a darkness object, and the transmission of pixel $x$ will be considered as unreliable. For each unreliable depth pixel, we use the transmission obtained by dark channel prior to measure its darkness. The pixel with smaller darkness weight should be attenuated more. Denote the transmission obtained by dark channel prior as $t(x)^{\text {dark }}$, then the unreliable transmission can be corrected as

$$
\tilde{t}(x)^{\text {corrected }}=t(x)^{\text {dark }} \times \tilde{t}(x)^{\text {bright }}
$$

Figure 6 presents one transmission estimation result via dark/bright channel prior from an input low illumination image (Fig. 6a) using the patch size $15 \times 15$. As can be seen in that part, missed structures in the transmission map estimated only via the bright channel prior (Fig. 6b) are observed via dark/bright channel prior (as indexed by red circle in Fig. 6c). However, though the transmission map is roughly good, there remains obvious block effects in some regions due to the primary operation of the dark channel prior depends on the minimum filter; the transmission map will usually experience a loss of edge information during estimation. For this reason, in the next subsection, we propose a refined transmission procedure that uses guided filter technique to preserve edge information of input images and thereby avoid generation of halo effects.

\subsection{Transmission map refinement using guided filter}

For the refining of the transmission map, there are many methods that can be used. For example, He used a soft matting algorithm to refine the transmission in [16]. However, the soft matting is a time-consuming technique. As an alternative, median filtering [24], bilateral filter [25], and guided filter [26] are used instead of soft matting. In this paper, considering both the time reduction and structure preservation, we choose the guided filter for refining

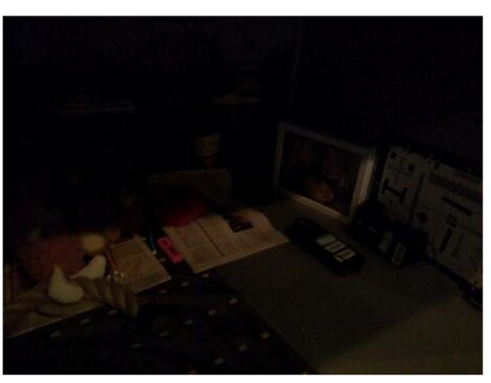

a

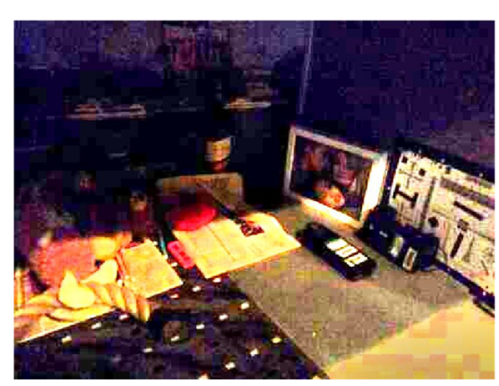

b

Fig. 8 Result of recovered scene radiance. a Original image. $\mathbf{b}$ Final recovered scene radiance 

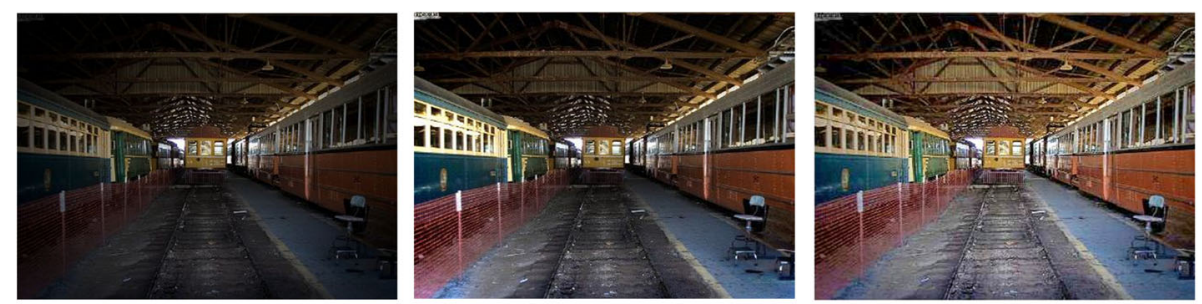

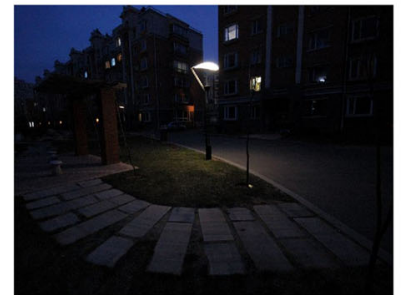

a

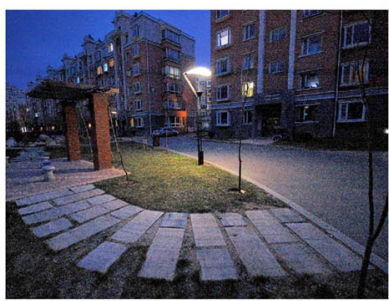

b

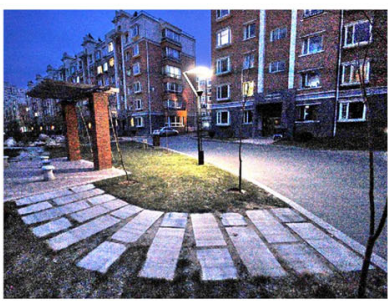

C

Fig. 9 Comparison of the proposed method with bright channel prior-based method [21]. a Input images. b Enhanced images with bright channel prior-based method [21]. c enhanced image with the proposed method

the transmission map. It should be noticed that though the main ideas used here is similar to that in [26], the meaning of transmission in this work is different from that in [26]. In [26], the transmission indexes the degree that light pass through the haze; whereas in this work, the transmission indexes the degree that light has been absorbed by air.

The main idea of guided filter is to filter input images by considering the content of the guidance image. Formally, given a guidance image $I$, a guided filtering output image $I_{\text {in }}^{\text {guided }}$ of input image $I_{\text {in }}$ can be defined as

$$
I_{\text {in }}^{\text {guided }}(x)=a_{k} I_{i}(x)+b_{k}, \forall i \in \omega_{k}
$$

where $\omega_{k}$ is a window centered at the pixel $x$ and $a_{k}$ and $b_{k}$ are assumed to be constant in $\omega_{k}$ and determined as

$$
\begin{aligned}
& a_{k}=\frac{\frac{1}{n_{\omega}} \sum_{i \in \omega_{k}} I_{i n}^{i} p_{i}-\mu_{k} p_{k}}{\sigma_{k}^{2}+\varepsilon} \\
& b_{k}=\bar{p}_{k}-a_{k} \mu_{k}
\end{aligned}
$$

where $\mu_{k}$ and $\sigma_{k}^{2}$ are the average value and the variance of the input image $I_{i} n$ in window $\omega_{k}$, respectively. $n_{\omega}$ is the number of pixels in window $\omega_{k} \cdot \bar{p}_{k}$ is the mean of the guided image in window $\omega_{k} . \varepsilon$ is the regularization parameter which is used to determine the intensity of the changed pixels.
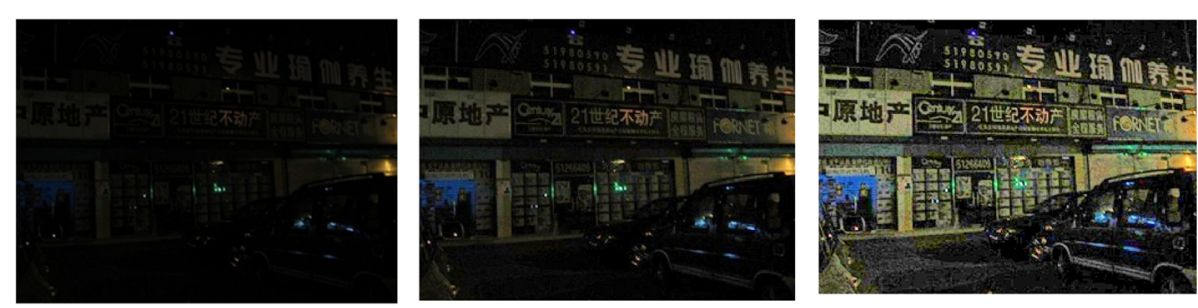

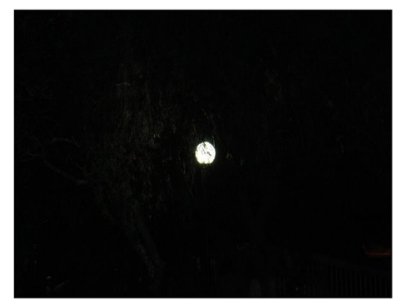

a

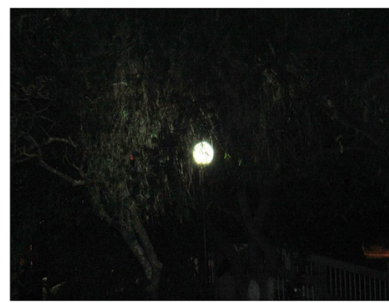

b

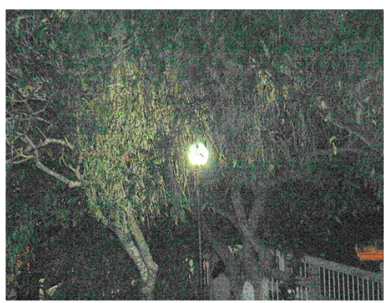

C

Fig. 10 Comparison of the proposed method with negative imaging dark channel-based method [20]. a Input images. b Enhanced images with negative imaging dark channel-based method [20]. c Enhanced image with the proposed method 

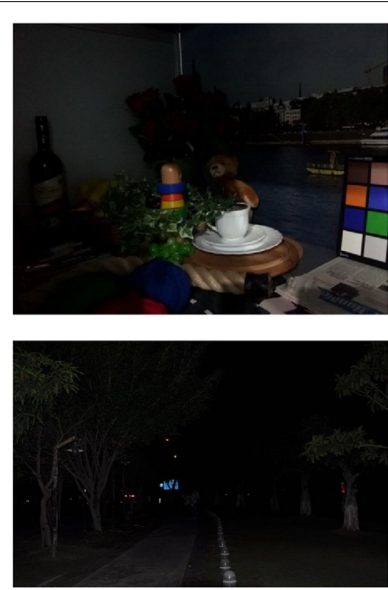

a
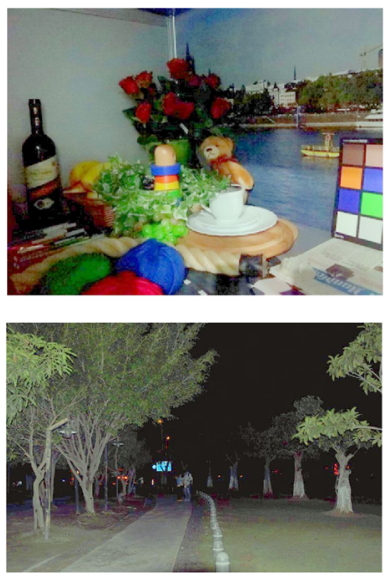

b
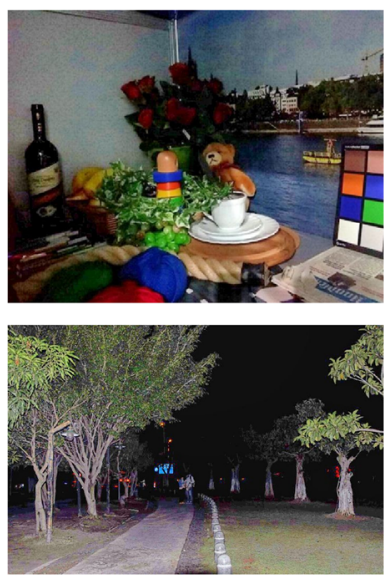

C

Fig. 11 Comparison of the proposed method with customized sigmoid function-based multi-scale retinex (MSR) [11]. a Input images. b Enhanced images with customized sigmoid function-based multi-scale retinex (MSR) [11]. c Enhanced image with the proposed method

Denote the refined transmission map by $t(x)$. The optimal $t(x)$ can be obtained as

$$
t(x)=a_{k} \tilde{t}^{\text {corrected }}(x)+b_{k}, \forall i \in \omega_{k}
$$

where $\tilde{t}^{\text {corrected }}$ is the transmission estimated by dual channel prior, definitions of parameter $a_{k}, b_{k}$, and $\omega_{k}$ are the same as what aforementioned.

Figure $7 \mathrm{c}$ shows the refined transmission with guided filter. As we can see from Fig. 7, in respective to the transmission estimated with dual channel prior, the refined transmission map manages to capture the sharp edge discontinuities and outline the profile of the objects.

\subsection{Recovering the scene radiance}

Once the atmospheric light $A$ and the final transmission map $t(x)$ are estimated, we can recover the scene radiance according to Eq. (4). However, we notice that, in Eq. (4), when the transmission $t(x)$ is close to zero, the term $\frac{I(x)-A}{t(x)}$ would be a very large value, and the directly recovered scene radiance $J$ would be noised. In order to avoid such a problem, we restrict the transmission $t(x)$ to a lower bound $t_{0}$. In this paper, the value of $t_{0}$ is 0.1 , which is determined according to experiments analysis. The final scene radiance $J(x)$ is recovered by

$$
J(x)=\frac{I(X)-A}{\max \left(t(x), t_{0}\right)}+A
$$

Figure $8 \mathrm{~b}$ shows the final recovered scene radiance by our method.
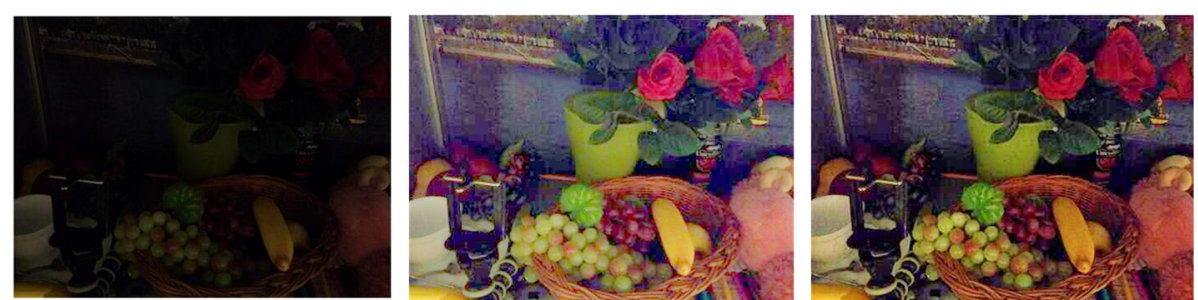

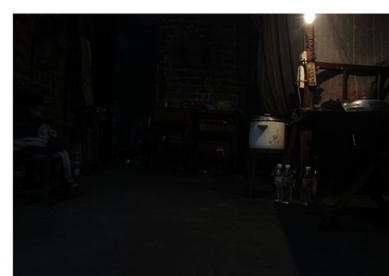

a

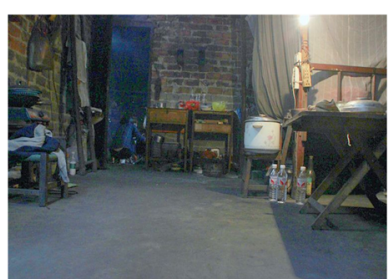

b

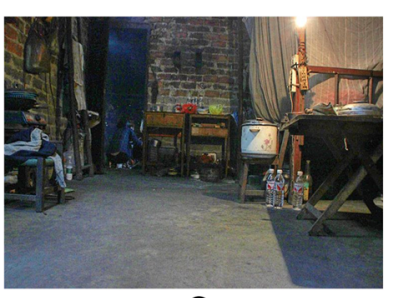

C

Fig. 12 Comparison of the proposed method with multi-scale retinex with color restoration (MSRCR) [13]. a Input images. b Enhanced images with multi-scale retinex with color restoration (MSRCR) [13]. c Enhanced image with the proposed method 

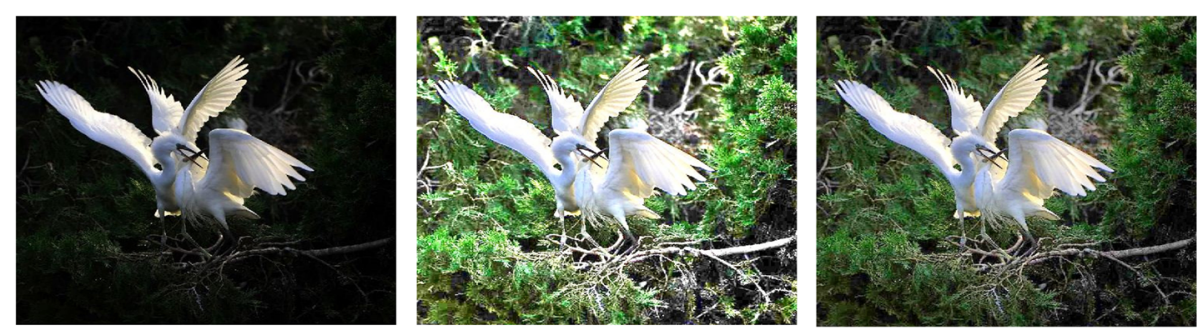

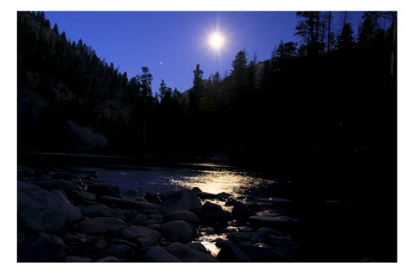

a

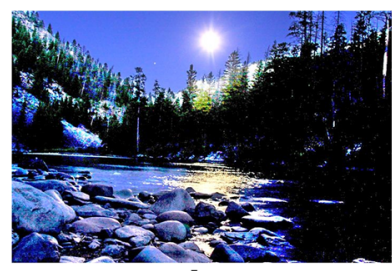

b

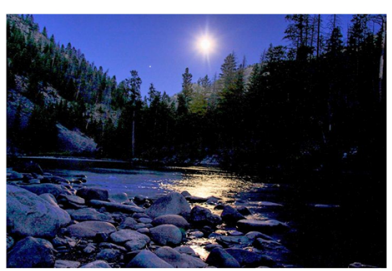

C

Fig. 13 Comparison of the proposed method with LIME [23]. a Input images. b Enhanced images with LIME [23]. c Enhanced image with the proposed method

\section{Experimental results}

\subsection{Experiment setting}

To evaluate the proposed method, extensive tests have been done using Matlab R2015 on a PC with a $2.60 \mathrm{GHz}$ Intel Pentium Dual Core Processor and 4G RAM.

For quantitative evaluation, an overall quality index proposed in [27] was employed in this paper. This indicator estimates the average visibility enhancement obtained by the restoration algorithm. The higher the overall quality index value is, the better the enhanced visibility is.

To the best of our knowledge, no standard nighttime low illumination image dataset is currently available for benchmarking. Hence, we collected totally 200 natural nighttime low illumination images from the Internet.

We compare the proposed method with six of the state of the art methods, including customized sigmoid function based multi-scale retinex (MSR) [11], multi-scale retinex with color restoration (MSRCR) [13], negative imaging dark channel-based method [20], bright channel prior-based method [21], and LIME [23].

The major parameters of the compared methods are consistent with the original papers and set as follows: for MSR, patch sizes of the three Gaussian functions are set to 15,80 , and 250, respectively . For dark channel [20], bright channel [21], LIME [23], and our method, the patch size $\Omega$ is assigned as $15 \times 15$; initial transmission coefficient $t_{0}$ is set as 0.1 . The threshold $\alpha$ in the proposed method is set as 0.4 , which came from experience prior.

\subsection{Qualitative results}

Figures 9, 10, 11, 12, 13, 14, and 15 show part comparison of the proposed method with bright channel prior-based method [21], negative imaging dark channelbased method [20], multi-scale retinex (MSR) with customized sigmoid function [11], multi-scale retinex with color restoration (MSRCR) [13], and LIME [23].

In Figs. 9 and 10, we compared the proposed method with bright channel prior-based method [21] and negative imaging dark channel-based method [20], respectively. It can be seen that all methods can enhance the contrast of the input image. However, details in dark regions in enhanced image with the proposed method (Figs. 9c and 10c) are more clearer than that with the bright channel prior-based method [21] (Fig. 9b) and negative imaging dark channel-based method [20] (Fig. 10b).

Figures 11 and 12 show comparison of the proposed method with multi-scale retinex (MSR) with customized sigmoid function [11] and multi-scale retinex with color restoration (MSRCR) [13], respectively.

From Fig. 11, it can be seen that there are obvious artifacts in the edge of regions of the enhanced image with customized sigmoid function-based multi-scale retinex. For example, the color of the plate in Fig. 11b (top row) is too bright to see the outline border of the white plate, and the color of leaves of the tree in Fig. 11b (bottom row) looks a little gray. Whereas, these artifacts are obviously eliminated in the enhanced image with the proposed method Fig. 11c, where details are highlighted, the image is brighter, and the color looks more lively and natural.

From Fig. 12, it can be seen that MSRCR algorithm gives a good color restoration, the color of the enhanced image is vivid, and the contrast and illumination of dark regions are also enhanced; invisible details in these dark regions become visible. However, the contrast of brighter regions degraded, and details look blur. For example, the flower in the face of the electric cooker in 

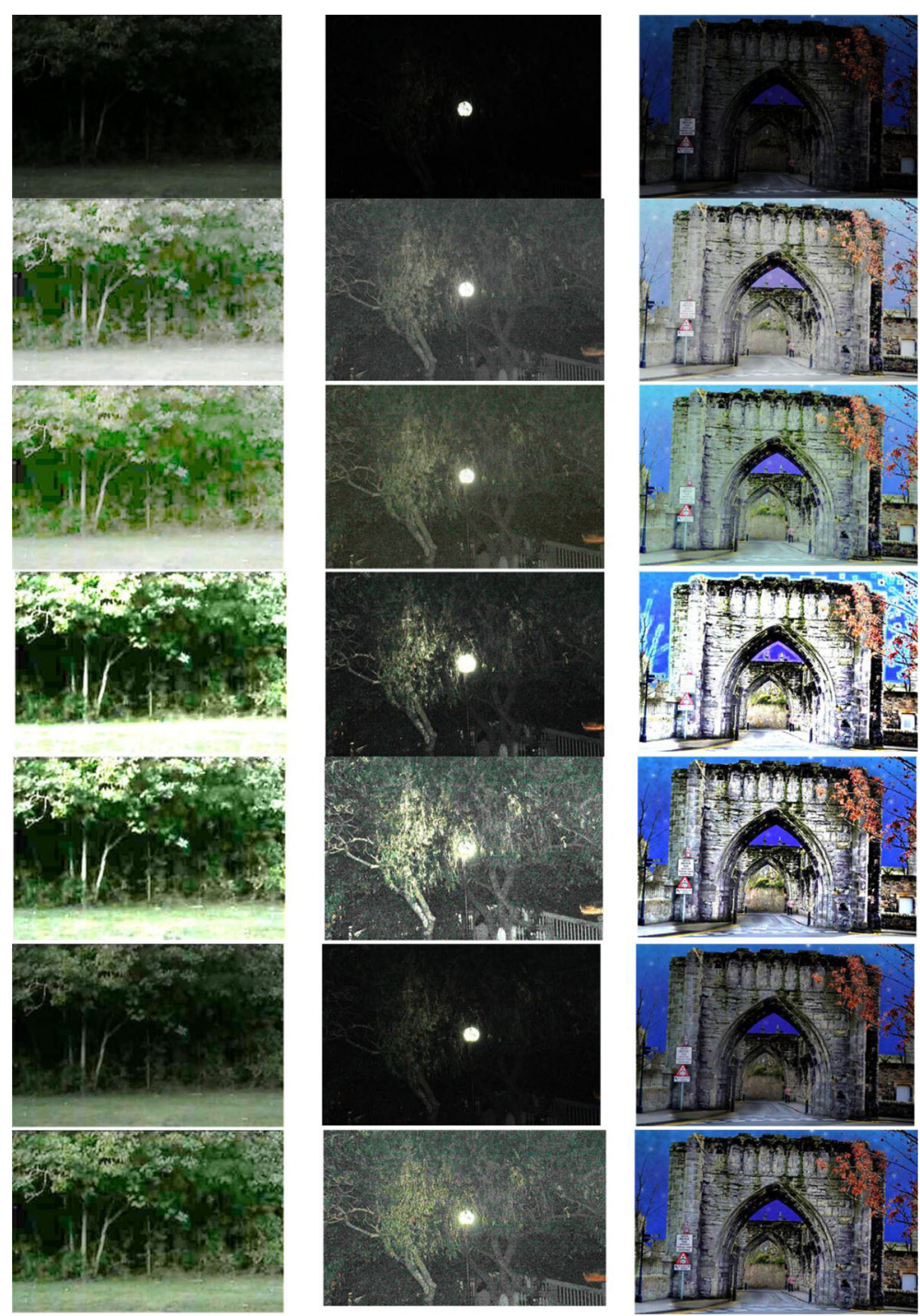

Fig. 14 Comparison of the proposed method with different methods. From top to bottom are original image, results with MSR [1 1], results with MSRCR [13], results with bright channel prior [21], LIME [23], results with negative dark channel [20], and that with the proposed method

Fig. 12b (bottom row) is difficult to see, while that in Fig. 12c (bottom row) is very clearly.

Figure 13 shows a comparison of the proposed method with LIME [23], one of the latest state of the art method for low illumination image enhancement. From Fig. 13, it can be seen that the image enhanced with LIME is brighter than that with the proposed method; however, the color of some regions looks unnatural, especially in bright white regions. There also an introduction the "halo" effect, for example in the top end of trees in Fig. 13b (bottom row). While the enhanced image with the proposed method looks very natural both in color and in visual (Fig. 13c).

Figure 14 shows an overview comparison of the proposed method with customized sigmoid function based multi-scale retinex (MSR)[11], multi-scale retinex with color restoration (MSRCR) [13], negative imaging dark channel-based method [20], bright channel prior-based method [21], and LIME [23]. It can be seen that the contrast of all input images (the first row in Fig. 14) are very low and the color are non-vivid. In respect to the input images, the illuminations of all images enhanced with the aforementioned methods have improved in different levels. Invisible details in the dark regions of the input image are all almost visible in the enhanced images. However, all bright channel-based methods including the method in [21] (the fourth row in Fig. 14) and LIME in [23] (the fifth row in Fig. 14) have obvious effect on improving the white brightness of the images and cause artifacts in the brighter regions. The MSR-based methods are not sensitive to white brightness; however, there is a serious 

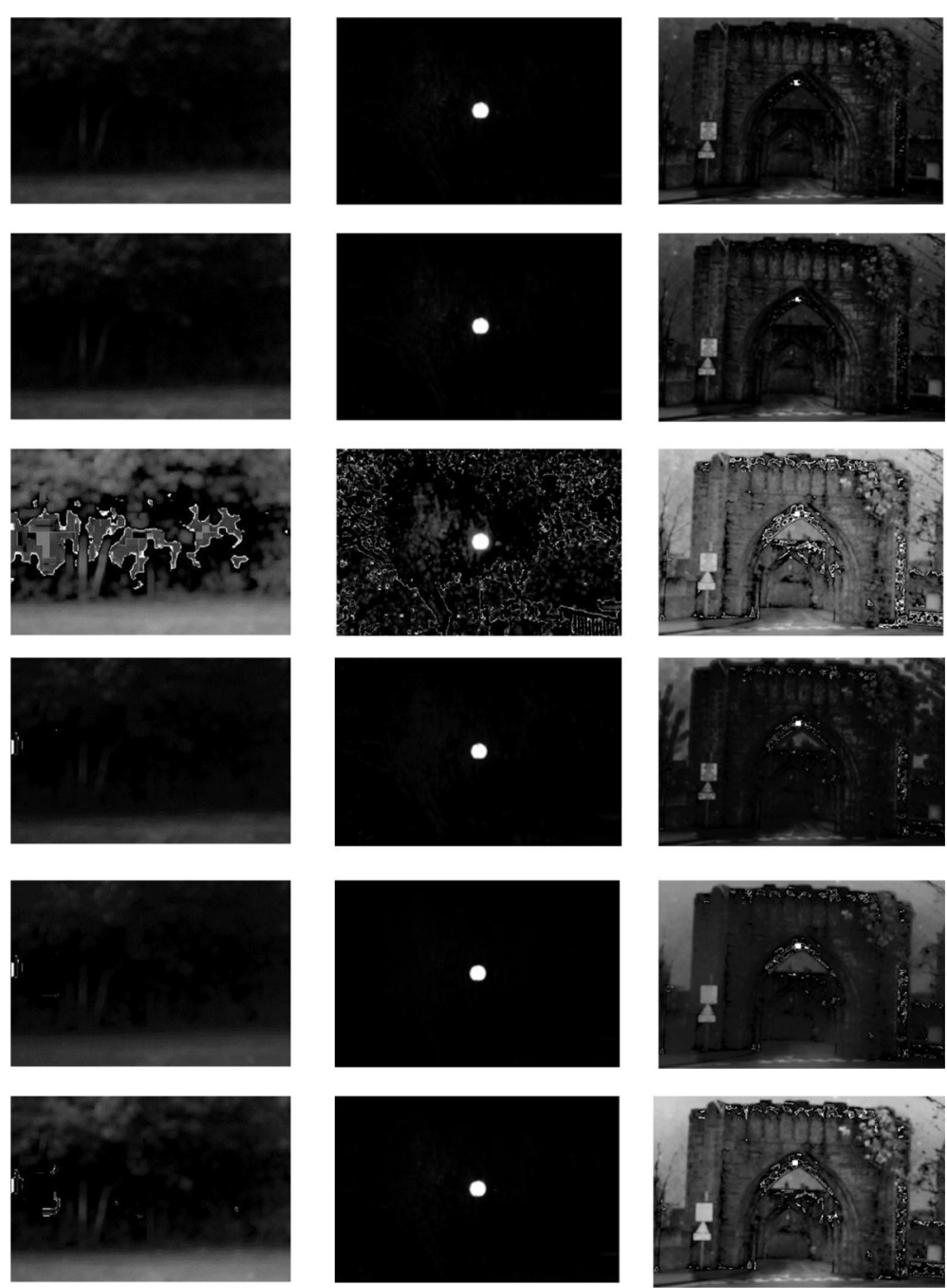

Fig. 15 The quality maps of different methods on the three images in Figure 12. From top to bottom are map for MSR [11], map for MSRCR [13], map for negative dark channel [20], map for bright channel prior [21], map for LIME [23], and that for proposed method

color distortion in the results with MSR [11] (the second row in Fig. 14), where the color is not vivid and looks a little dimmer. Though the color of the enhanced image with MSRCR is corrected, the brightness of the enhanced image looks unnatural. The visual of the enhanced images with the negative dark channel-based method [20] (the six row in Fig. 14) looks good, but the image is a little dark and the details in the dark regions are not clear to be seen. In restoring the invisible details in dark regions, the proposed method is obviously superior to all other aforementioned methods; as shown in the last row in Fig. 14, invisible details in dark regions and bright regions are well restored, which avoids detail blur caused by overenhancement. Halo artifacts are also obviously alleviated.

\subsection{Quantitative analysis}

Table 1 shows a comparison of the aforementioned different methods on a large amount of nighttime low illumination images in terms of the average overall quality [27]. From Table 1, it can be seen that the average overall quality index values of the proposed method are much higher than that of other methods, which indicates that the proposed method remarkably improves the visibility

Table 1 Comparison of the average overall quality index of different methods

\begin{tabular}{ll}
\hline Methods & The average overall quality index \\
\hline MSR [11] & 0.0576 \\
MSRCR [13] & 0.0637 \\
$\begin{array}{l}\text { Negative imaging dark channel } \\
\text { based method [20] }\end{array}$ & 0.1278 \\
$\begin{array}{l}\text { Bright channel prior based method } \\
\text { [21] }\end{array}$ & 0.0470 \\
LIME [23] & 0.0720 \\
Proposed method & 0.1589
\end{tabular}


Table 2 Comparison of the average computation time of different methods

\begin{tabular}{ll}
\hline Methods & The average computation time (s) \\
\hline MSR [1 1] & 0.5398 \\
MSRCR [13] & 0.8767 \\
$\begin{array}{l}\text { Negative imaging dark channel } \\
\text { based method [20] }\end{array}$ & 0.5449 \\
$\begin{array}{l}\text { Bright channel prior based method } \\
\text { [21] }\end{array}$ & 0.5336 \\
LIME [23] & 0.5368 \\
Proposed method & 0.6842
\end{tabular}

of the images compared to other methods. From Table 1, we also noticed that the overall quality index values of the negative imaging dark channel-based method [16] and that of the proposed method are closer and both are the higher and are 0.1278 and 0.1589 , respectively. In fact, if visibility restoration algorithms must increase the contrast, artificial edges must not become visible. Figure 15 shows the quality maps of different methods on the three images in Fig. 14. From the maps, it can be seen that the map with the proposed method has clear and smoothing image structure for all the images in Fig. 14, whereas other

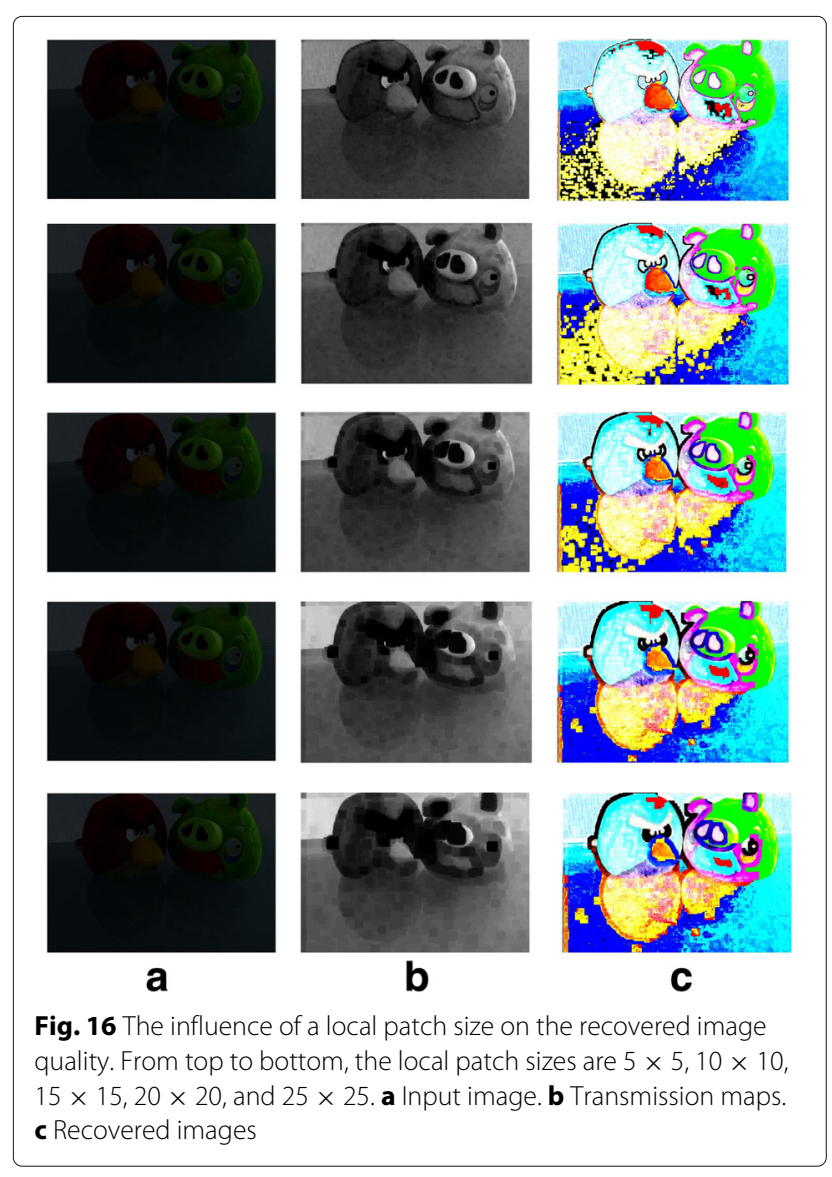

methods do not, and this is consistent with what we see from Table 1 and observed in Fig. 14.

Table 2 shows the average computation time of the aforementioned method. From Table 2, it can be seen that in the view of the computation time, the proposed method is not the best method; of all the six methods, the computation time of the proposed method is only shorter than that of MSRCR [13], which is $0.6842 \mathrm{~s}$. In this view, there is space for improving the performance of our method in the future.

Simply, in this work, we assume that the transmission in a local patch $\Omega(x)$ is a constant. However, as we all know that in fact, the transmission is not a constant, we also evaluate the influence of the size of the local patch $\Omega(x)$ on the processing results. Figure 16 shows the influence of a local patch size on the recovered image quality. As can be seen that with the local patch size becoming from $5 \times 5$ to $25 \times 25$, the image recovered more better. For example, for patch size $5 \times 5$, the transmission map looks a little dim, and there are many obvious noises in the recovered image; whereas, with the increase of the patch size, the transmission map becomes more bright, where the image structure is more clear, and there is little noise in the recovered image since the patch size became $15 \times 15$. However, we also found that with the increase of a local patch size, more time is needed to estimate the transmission, as shown in Table 2. Based on afore discussion, in this paper, considering both of the recovered image quality and computing time, we select a local patch size of $15 \times 15$.

\section{Discussion}

As mentioned in Section 4.4, the transmission map usually experiences a loss of edge information when estimation due to the primary operation of the bright/dark channel prior depends on the maximum/minimum filter. To handle this problem, we propose a refined transmission procedure. Figure 17 shows the comparison of the effect of different transmission optimization method on the recovered image quality. From Fig. 17, it can be seen that the transmission optimized with guided filter has the most smoothing image structure; the details are also clearer than that with other methods. Transmission map optimized with softmap looks darker, and the image structure is not clear. For recovered image quality, it can be seen that both recovered images with bilateral filter and guided filter have good recovery results. The recovered image with softmap looks a little dark, and the details in the dark regions are not clear. Table 3 shows the running time for the different transmission optimization methods on a $510 \times 729$ image. It can be seen that it only takes $0.331529 \mathrm{~s}$ to optimize the initial transmission with guided filter, while that with the bilateral filter is $12.745949 \mathrm{~s}$, and that with the softmap method is $31.404145 \mathrm{~s}$. Based on afore discussions, in 

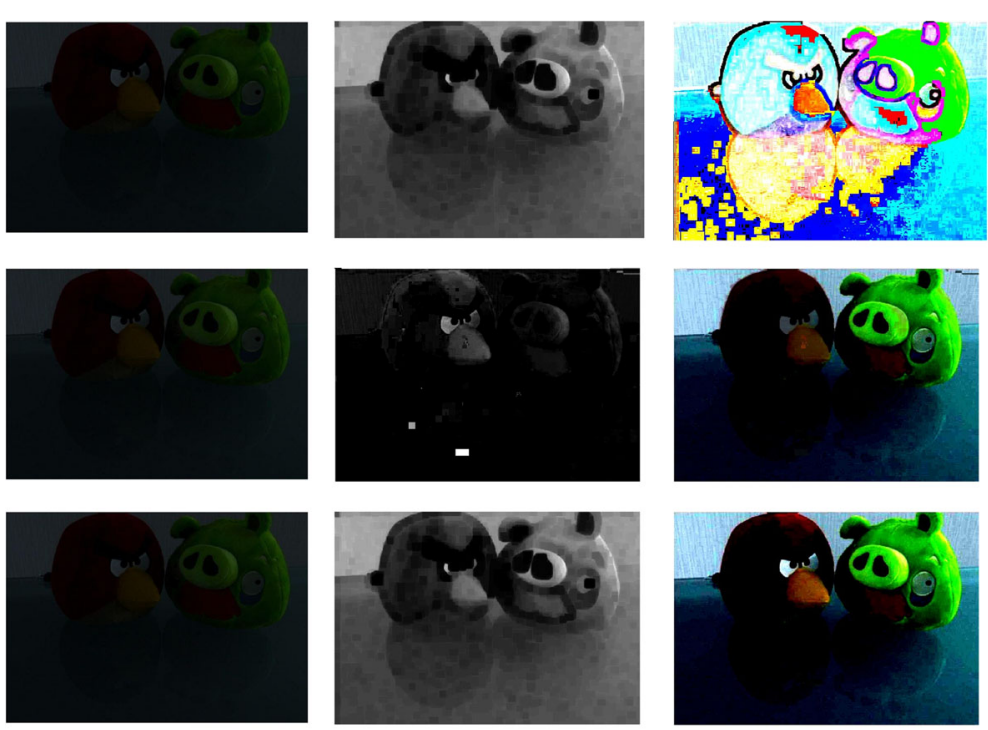

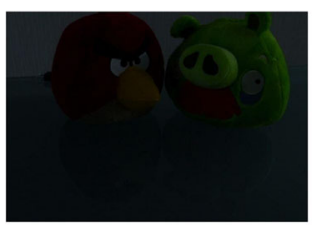

a

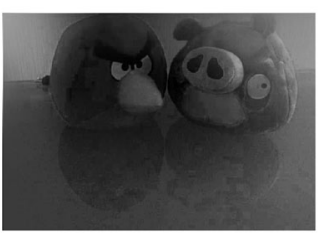

b

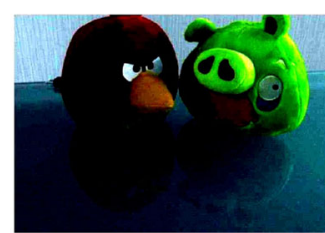

C

Fig. 17 Comparison of the effect of different transmission optimization methods on the recovered image quality. From top to bottom, non-optimized transmission, softmap-optimized transmission, bilateral filtering transmission, and guided filtering transmission. a Input image. b Transmission maps. c Recovered images

this paper, we use guided filter to optimize the initial transmission.

\section{Conclusions}

In this paper, a dual channel prior-based method for low illumination image enhancement with a single image is proposed, which builds upon two existing image priors: dark channel prior and bright channel prior, where we utilize the bright channel prior to get an initial transmission estimate and then use the dark channel as a complementary channel to correct potentially erroneous transmission estimates attained from the bright channel prior. We evaluated the performance of the proposed method in terms

Table 3 The influence of a local patch size on transmission estimation time (image size $510 \times 729$ )

\begin{tabular}{ll}
\hline Patch size & Time $(\mathrm{s})$ \\
\hline $5 \times 5$ & 1.359072 \\
$10 \times 10$ & 1.503148 \\
$15 \times 15$ & 1.771014 \\
$20 \times 20$ & 2.482208 \\
$25 \times 25$ & 2.074232 \\
\hline
\end{tabular}

of qualitative analysis and quantitative analysis. Experiments on a large quantity of low illumination images revealed that the proposed method generated very highquality enhancement images compared with the other state-of-the-art methods.

As demonstrated by the experimental results, the main advantage of the proposed method can obtain more details compared with dark channel prior- or bright channel prior-based method. However, it still needs to be further improved. For example, to avoid the halo effect, the size of the local patch for both dark channel prior and bright channel prior needs to be set manually, but it is an experience-based work. Another drawback is its expensive computation complexity. As we mentioned in the experiment section, in terms of computation time, our method is not the best one; therefore, in the future, we

Table 4 Running time for different transmission optimization methods on a $510 \times 729$ image

\begin{tabular}{ll}
\hline Method & Time (s) \\
\hline Soft-map & 31.404145 \\
Bilateral filter & 12.745949 \\
Guided filter & 0.331529
\end{tabular}


will consider how to choose the size of local patch adaptively. We will also focus on reducing the running time of the method in order to make it meet the need of in real applications.

\section{Acknowledgements}

The authors thank the editor and anonymous reviewers for their helpful comments and valuable suggestions.

\section{Funding}

This work was supported in part by a grant from the National Natural Science Foundation of China (No. 61202198, No. 61401355, No. 41601353), a grant from the China Scholarship Council (No. 201608610048), the Key Laboratory Foundation of Shaanxi Education Department (No. 14JS072), and the Nature Science Foundation of Science Department of PeiLin count at Xi'an(GX1619).

\section{Availability of data and materials}

The datasets used and/or analysed during the current study are available from the corresponding author on reasonable request.

\section{Authors' contributions}

All authors take part in the discussion of the work described in this paper. The author ZS wrote the first version of the paper. The author ZM did part experiments of the paper, ZM, GZ and CZ revised the paper in different version of the paper, respectively. The contributions of the proposed work are mainly in two aspects: (1) We propose an accuracy transmission estimation method based on bright channel prior and dark channel prior. We firstly utilize the bright channel prior to get an initial transmission estimation and then use the dark channel as a complementary channel to correct potentially erroneous transmission estimates attained from the bright channel prior. (2) We develop an effective method for low illumination image enhancement with a single image based on bright channel prior and dark channel prior. All authors read and approved the final manuscript.

\section{Authors' information}

\section{Author1}

Zhenghao Shi received the BS degree in Material Science and Engineering from Dalian Jiaotong University, Dalian, China, in 1995, the MS degree in Computer Application Technology from Xi'an University of Technology, Xi'an, China, in 2000, and the Ph.D degree in Computer Architecture from Xi'an Institute of Microelectronics, Xi'an, China, in 2005. In 2000, he joined Xi'an University of Technology, Xi'an, China. From 2000 to 2005, he was an assistant professor in the Department of Computer Science and Engineering at Xi'an University of Technology. From 2006 to now, he is an associate professor of the Department of Computer Science and Engineering at the same university. Since December 2016, he joined IDBE laboratory, University of North Carolina at Chapel Hill as a visiting associate professor. During the period of 2006 to 2007 and of 2008 to 2009, he was on leave with the department of computer science and engineering at Nagoya institute of technology, Nagoya, Japan, for image research as a postdoctral researcher, respectively. From 2007 to 2008, he was a research associate in the Kurt Rossmann Laboratories for Radiologic Image Research, the Department of Radiology, the Division of Biological Sciences, the University of Chicago. His research interests include neural networks for image processing and pattern recognition, computer-aided diagnosis, and image processing suggested by the human visual systems. He is a member of the IEEE and also a member of ACM.

Contact: zhenghaoshimtap@163.com

\section{Author2}

Meimei Zhu is currently studying for her master degree in Computer Science and Technology in Xi'an University of Technology, Xi'an, China. Her research interests focus on image processing.

Contact:1256260733@qq.com

\section{Author3}

Bin Guo is currently a professor with Northwestern Polytechnical University, China. He was previously a post-doctoral researcher of Prof. Daqing Zhang's research group at Institute TELECOM SudParis, France. He received his Ph.D. degree in Computer Science from Keio University, Tokyo, Japan, in 2009. He finished his Ph.D studies in the Anzai-Imai Lab, under the supervision of Prof. Michita Imai and Prof. Yuichiro Anzai (president of JSPS in Japan). He received his B.E and M.E of Computer Science from Xi'an Jiaotong University (in China), in 2003 and 2006, respectively. His current research interests include the following: Ubiquitous Computing, Social and Community Intelligence, Urban Big Data Mining, Mobile Crowd Sensing, and Human-Computer Interaction. He has served as the general co-chair of the 12th IEEE International Conference on Ubiquitous Intelligence and Computing (IEEE UIC'15) and the program chair of IEEE CPSCom'16, IEEE UIC'13, the general chair of the IEEE 2014 International Workshop on Social and Community Intelligence (IEEE SCl'14@PerCom'14), the program vice-chair of the The 5 th International Conference on Ambient Systems, Networks and Technologies (ANT'14) and the 14th IEEE International Conference on Computer and Information Technology (IEEE CIT'14), and so on. He serves as the TPC member for a number of conferences, including UbiComp, PerCom, MobiQuitous, MoMM, GPC, ICOST, EUC, etc. He is a senior member of IEEE and CCF, a member of ACM, a Technical Committee member of Big Data at IEEE Communications Society, CSCWD at IEEE SMC Society, and CCF Prevasive Computing Group. He has published over 100 papers in refereed journals, conference proceedings, and book chapters. Contact: guob@nwpu.edu.cn

\section{Author4}

Minghua Zhao received her Ph.D degree in Computer Science from Sichuan University, Chengdu, China, in 2006. After that, she joined Xi'an University of Technology, Xi'an, China. Currently, she is an associate professor of the Department of Computer Science and Engineering at the same university. Her research interests include image processing and pattern recognition. Contact: zhaominghua@xaut.edu.cn

\section{Author5}

Changqing Zhang received his B.S. and M.S. degrees from the College of Computer Science, Sichuan University in 2005 and 2008, respectively and the Ph.D. degree in Computer Science from Tianjin University in 2016. He is an Assistant Professor at the School of Computer Science and Technology, Tianjin University. His current research interests include machine learning, data mining, and computer vision.

Contact: zhangchangqing@tju.edu.cn

\section{Competing interests}

There are no potential competing interests in our paper.

\section{Publisher's Note}

Springer Nature remains neutral with regard to jurisdictional claims in published maps and institutional affiliations.

\section{Author details}

${ }^{1}$ School of Computer Science and Engineering, Xi'an University of Technology, No.5 Jinhua South Road, 710048 Xi'an, China. ${ }^{2}$ School of Computer Science and Engineering, Northwestern Polytechnical University, No.127 West Youyi Road, 710072 Xi'an, China. ${ }^{3}$ School of Computer Science and Technology, Tianjing University, No.135 Yaguan Road, 300350 Tianjin, China.

Received: 19 October 2017 Accepted: 29 January 2018

Published online: 14 February 2018

\section{References}

1. GR Vishalakshi, MT Gopalakrishna, MC Hanumantharaju, in Comprehensive review of video enhancement algorithms for low lighting conditions, ed. by S Satapathy, J Mandal, S Udgata, and V Bhateja. Advances in Intelligent Systems and Computing (Springer, New York, NY, 2016), pp. 475-485

2. S Yin, L Cao, Y Ling, G Jin, One color contrast enhanced infrared and visible image fusion method. Infrared Phys Technol. 53, 146-150 (2010)

3. MA Hogervorst, A Toet, Fast natural color mapping for night-time imagery. Inf Fusion. 11, 69-77 (2010)

4. VTsagaris, V Anastassopoulos, Fusion of visible and infrared imagery for night color vision. Displays. 26, 191-196 (2005)

5. Q Xiaoyan, Y Wang, B Wang, Fast color contrast enhancement method for color night vision. Infrared Physics \& Technol. 55, 122-129 (2012)

6. MS Sayed, J Delva, in Low complexity contrast enhancement algorithm for low illumination visual surveillance, ed. by Aboul Ella Hassanien, FMHHMAT-PH, and Ajith Abraham. Proceedings of the 2010 10th 
International Conference on Intelligent Systems Design and

Applications:November 29-December 1, 2010;Cairo, Egypt, (Egypt, 2010), pp. 835-838. Special Session, Advances in Robotics \& VR

7. Z Zhou, N Sang, X Hu, Global brightness and local contrast adaptive enhancement for low illumination color image. Optik. 125, 1795-1799 (2014)

8. SCF Lin, CY Wong, MA Rahman, G Jiang, S Liu, N Kwok, H Shi, Y-H Yu, $\mathrm{T}$ Wu, Image enhancement using the averaging histogram equalization (AVHEQ) approach for contrast improvement and brightness preservation. Comput Electr Eng. 46, 356-370 (2015)

9. TL Kong, NAM Isa, Enhancer-based contrast enhancement technique for non-uniform illumination and low-contrast images. Multimed. Tools Appl. 76, 14305-14326 (2017)

10. C Jung, Q Yang, T Sun, Q Fu, H Song, Low light image enhancement with dual-tree complex wavelet transform. J Vis Commun Image R. 42, 28-36 (2017)

11. H Lin, Z Shi, Multi-scale retinex improvement for low illumination image enhancement. Optik. 125, 7143-7148 (2014)

12. H Kuang, $\mathrm{L}$ Chen, $\mathrm{F}$ Gu, J Chen, $\mathrm{L}$ Chan, Combining region-of-interest extraction and image enhancement for low illumination vehicle detection. IEEE Intell Syst. 31, 57-65 (2016)

13. I-S Jang, H-G Ha, T-H Lee, Y-H Ha, Color correction by estimation of dominant chromaticity in multi-scaled retinex. J Imaging Sci Technol. 53, 501-512 (2009)

14. K Fotiadou, G Tsagkatakis, P Tsakalides, in Low light image enhancement via sparse representation, ed. by A Campilho, M Kamel. Image Analysis and Recognition (Springer, Switzerland, 2014), pp. 12-19

15. H Fu, H Ma, S Wu, in 21 st International Conference on Pattern Recognition (ICPR 2012):11-15 Nov. 2012;Tsukuba, Japan. Night removal by color estimation and sparse representation, (Japan, 2012), pp. 3656-3659. the International Association for Pattern Recognition

16. J He, K Sun, X Tang, Single image haze removal using dark channel prior. IEEE Transactions on Pattern Analysis and Machine Intelligence. 33, 2341-2353 (2011)

17. Y Gong, Y Lee, TQ Nguyen, in 2016 International SoC Design Conference:October 23-26, 2016; Jeju, Korea. Low illumination image enhancement applying dark channel prior to raw data from camera (IEEE, 2016), pp. 173-174

18. B Feng, $Y$ Tang, $L$ Zhou, $Y$ Chen, in 2016 8th International Conference on Wireless Communications \& Signal Processing:13-15 Oct. 2016; Yangzhou, China. Image enhancement under low luminance with strong light weakening (Nanjing University of Posts and Telecommunications, 2016), pp. 1-5

19. J Song, L Zhang, P Shen, X Peng, G Zhu, in Chinese Conference on Pattern Recognition, CCPR 2016:November 5-7, 2016; Chengdu, China, ed. by Tieniu Tan, XuelongLi XCJZJY, and H Cheng. Single low-light image enhancement using luminance map (Dianzi keji Unviersity, 2016), pp. 101-110

20. Z Shi, M Zhu, B Guo, M Zhao, A photographic negative imaging inspired method for low illumination night-time image enhancement. Multimed Tools Appl. 76, 15027-15048 (2017)

21. Y Wang, S Zhuo, D Tao, J Bu, N Li, Automatic local exposure correction using bright channel prior for under-exposed images. Signal Processing 93, 3227-3238 (2013)

22. H Cho, H Lee, S Lee, in Computer Vision-ECCV 2014:September 6-12, 2014: Zurich, Switzerland, ed. by D. e. a. Fleet. Radial bright channel prior for single image vignetting correction (swissICT, 2014), pp. 189-202

23. X Guo, Y Li, H Ling, LIME: Low-light image enhancement via illumination map estimation. IEEE Transactions on Image Processing. 26, 982-993 (2017)

24. KB Gibson, DT Vo, TQ Nguyen, An investigation of dehazing effects on image and video coding. IEEE Transactions on Image Processing. 21, 662-673 (2012)

25. CYu, J Xiao, D Li, in Proceedings of the IEEE 10th International Conference on Signal Processing. Physics-based fast single image fog removal (swisslCT, 2010), pp. 1048-1052

26. J He, K Sun, X Tang, Guided image filtering. IEEE Transactions on Pattern Analysis and Machine Intelligence. 35, 1397-1409 (2013)

27. Z Wang, AC Bovik, Universal image quality index. IEEE Signal Processing Letters. 9, 81-84 (2001)

\section{Submit your manuscript to a SpringerOpen ${ }^{\circ}$ journal and benefit from:}

- Convenient online submission

- Rigorous peer review

- Open access: articles freely available online

- High visibility within the field

- Retaining the copyright to your article

Submit your next manuscript at $\gg$ springeropen.com 